\title{
High-Order-Harmonic Generation and Superradiance in a Seeded Free-Electron Laser
}

\author{
L. Giannessi, ${ }^{1, *}$ M. Artioli, ${ }^{1}$ M. Bellaveglia, ${ }^{2}$ F. Briquez,${ }^{9}$ E. Chiadroni,${ }^{2}$ A. Cianchi, ${ }^{7}$ M. E. Couprie, ${ }^{9}$ \\ G. Dattoli, ${ }^{1}$ E. Di Palma, ${ }^{1}$ G. Di Pirro, ${ }^{2}$ M. Ferrario, ${ }^{2}$ D. Filippetto,${ }^{10}$ F. Frassetto, ${ }^{5}$ G. Gatti, ${ }^{2}$ M. Labat, ${ }^{9}$ \\ G. Marcus, ${ }^{8}$ A. Mostacci, ${ }^{4}$ A. Petralia, ${ }^{1}$ V. Petrillo,${ }^{3}$ L. Poletto,${ }^{5}$ M. Quattromini, ${ }^{1}$ J. V. Rau, ${ }^{6}$ \\ J. Rosenzweig, ${ }^{8}$ E. Sabia, ${ }^{1}$ M. Serluca, ${ }^{4}$ I. Spassovsky, ${ }^{1}$ and V. Surrenti ${ }^{1}$ \\ ${ }^{1}$ ENEA C.R. Frascati, Via E. Fermi, 4500044 Frascati, Roma, Italy \\ ${ }^{2}$ INFN-LNF, Via E. Fermi, 4000044 Frascati, Roma, Italy \\ ${ }^{3}$ Università degli Studi di Milano and INFN-Mi, Via Celoria, 1620133 Milano, Italy \\ ${ }^{4}$ Università La Sapienza, Piazzale Aldo Moro, 100185 Roma, Italy \\ ${ }^{5}$ IFN-CNR, Via Trasea 7, 35131 Padova, Italy \\ ${ }^{6}$ ISM-CNR Via del Fosso del Cavaliere,100-00133 Roma, Italy \\ ${ }^{7}$ Università di Roma Tor Vergata and INFN-Roma TV, Via della Ricerca Scientifica, 100133 Roma, Italy \\ ${ }^{8}$ UCLA, 405 Hilgard Avenue, Los Angeles, California 90095-1547, USA \\ ${ }^{9}$ SOLEIL, L'Orme des Merisiers Saint-Aubin - BP 4891192 GIF-sur-Yvette, Cedex, FR \\ ${ }^{10}$ Lawrence Berkeley National Laboratory, 1 Cyclotron Road, Berkeley, California 94720, USA
}

(Received 5 January 2012; published 19 April 2012)

\begin{abstract}
Higher order harmonic generation in a free-electron laser amplifier operating in the superradiant regime [R. H. Dicke, Phys. Rev. 93, 99 (1954).] has been observed. Superradiance has been induced by seeding a single-pass amplifier with the second harmonic of a Ti:sapphire laser, generated in a $\beta$-Barium borate crystal, at seed intensities comparable to the free-electron laser saturation intensity. Pulse energy and spectral distributions of the harmonics up to the 11th order have been measured and compared with simulations.
\end{abstract}

DOI: 10.1103/PhysRevLett.108.164801

PACS numbers: 41.60.Cr, 41.50.+h, 42.55.Vc

Nonlinear harmonic generation is widely used to extend the operation of optical lasers to the UV-vacuum ultra violet (UV-VUV) spectral region, where research methods for the investigation of matter [1-4] require ultrashort coherent pulses. Frequency up-conversion to the $10-100 \mathrm{~nm}$ range may be accomplished with high harmonics generated in gas $(\mathrm{HHG})[5,6]$, where the active medium is a low density noble gas. The emission of high-energy photons, however, is inherently coupled with ionization, and the use of a nonlinear optical medium poses limitations to the conversion efficiency at the shortest wavelengths. In the spectral region where ionization processes are dominant, harmonic generation may still be obtained in freeelectron lasers (FELs). The mechanism of frequency up-conversion is based on the nonlinear density modulation of an electron beam at a given seed wavelength, $\lambda_{\text {seed }}$. The frequency components of the modulated beam enforce the collective emission process at the resonant wavelength $\lambda_{r}=\lambda_{u}\left(1+K^{2} / 2\right) / 2 \gamma^{2} \approx \lambda_{\text {seed }}$ and its harmonics $[\gamma$ is the Lorentz factor of the electrons, $\lambda_{u}$ is the period, and $K=e B_{u} \lambda_{u} /\left(2 \pi m_{e} c\right)$ is the deflection parameter of the undulator] [7]. The seed transfers its longitudinal coherence properties to the FEL pulse [8], and schemes based on this principle were proposed to improve the longitudinal coherence of FELs operating in a self amplified spontaneous emission mode [9-11], where the temporal pulse structure is dominated by stochastic fluctuations associated with the initial shot noise [12]. The seed amplification, combined with the generation of coherent harmonics, has been demonstrated first in the midinfrared $[8,13]$ and then in the UV-VUV range [14-17]. User facilities based on the frequency up-conversion of a seed laser in the VUV soft-x-ray region of the spectrum are now in operation and provide radiation with unprecedented properties of longitudinal coherence [18]. Coherent harmonics have been observed when seeding a single-pass FEL amplifier, and include the 3rd, 5th [19], and 7th harmonics [20]. The process of harmonic generation is expected to extend to higher orders when the FEL operates in the regime of super-radiance [21], in which a short optical pulse slips over the electron beam and increases its energy while maintaining a self-similar shape [22,23]. In this regime, the radiation pulse has a peak power that increases with the square of the distance $z$ along the undulator and a longitudinal width that decreases with the inverse square root of $z$. The front of the pulse presents a peculiar structure, characterized by a modulation of the longitudinal phase space density with high harmonic content, as shown in Fig. 1. The self-similar nature of the radiation and particle system preserves this modulation, while the pulse propagates through the undulator, and the condition of phase matching is ensured by the fact that after saturation the pulse shifts over the electron beam at the velocity of light [24].

In this Letter, we present the experimental results on the generation of harmonics up to the 11th order in a seeded FEL operated in the superradiant regime. The experiment 


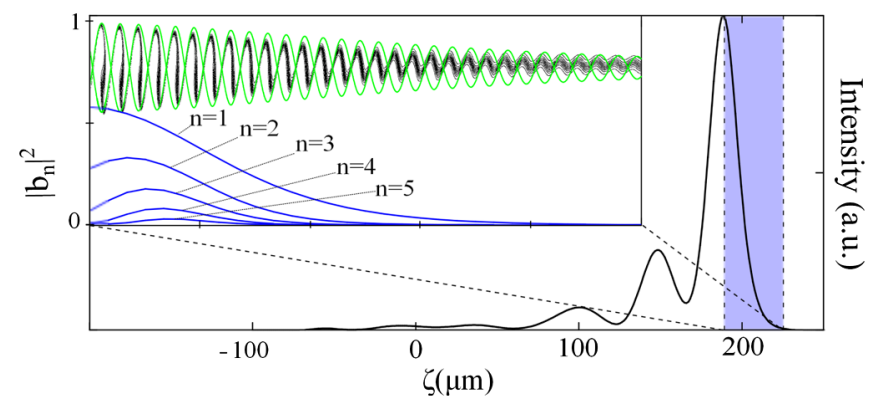

FIG. 1 (color online). Self-similar shape of a superradiant pulse along the $\zeta$ coordinate in the $e$-bunch frame at the end of the SPARC amplifier [PERSEO simulation [29] with the parameters in Table I, case (B)]. Insertion: Longitudinal phase space and bunching coefficients $\left|b_{n}\right|^{2}$ for the first 5 harmonics in the front side of the pulse (highlighted region).

was performed at the SPARC FEL facility [25] with the main electron beam parameters summarized in Table I. The data were collected during two different shifts, labeled as (A) and (B). The electron beam was injected in the undulator system, consisting of six sections of 75 periods each, with $\lambda_{u}=2.8 \mathrm{~cm}$ [26]. Superradiance was induced, as in the configuration tested in [23], by seeding the FEL as a single-pass amplifier, with a short laser pulse of peak power comparable to the FEL saturation intensity. The undulators were all tuned at $\lambda_{r}=\lambda_{\text {seed }}$ and the Twiss parameter $\langle\beta\rangle \simeq 1.5 \mathrm{~m}$ ensured the optimum beam matching.
TABLE I. Main parameters. The beam energy uncertainty is related to the pulse to pulse jitter and does not reflect the precision of the measurement.

\begin{tabular}{lcc}
\hline \hline & $(\mathrm{A})$ & $(\mathrm{B})$ \\
\hline Beam energy $E_{B}(\mathrm{MeV})$ & $172.18 \pm 0.27$ & $177.2 \pm 1.1$ \\
Beam charge $(\mathrm{pC})$ & $400 \pm 16$ & $460 \pm 18$ \\
Energy Spread $($ proj. $\%)$ & $0.11 \pm 0.015$ & $0.12 \pm 0.01$ \\
Energy Spread $($ slice \%) & $0.050 \pm 0.005$ & $0.050 \pm 0.005$ \\
Length r.m.s. $(\mathrm{ps})$ & $2.40 \pm 0.05$ & $2.65 \pm 0.049$ \\
Beam current $I_{\text {peak }}(A)$ & $58.0 \pm 3.5$ & $60.0 \pm 3.5$ \\
Vertical Emittance $(\mathrm{mm}$ mrad) & $3.0 \pm 1.0$ & $2.9 \pm 1.3$ \\
Horizontal Emittance $(\mathrm{mm}$ mrad) & $3.9 \pm 1.1$ & $3.2 \pm 1.3$ \\
Seed energy $(\mu \mathrm{J})$ & $0.0012 \div 2.0$ & 9.0 \\
Undulator deflection par. $K$ & 2.12 & 2.21 \\
\hline \hline
\end{tabular}

The seed laser system consists of a Ti:sapphire regenerative amplifier (Legend HFE by Coherent), driven by the same oscillator as the photocathode laser and delivers up to $2.5 \mathrm{~mJ}$ at $800 \mathrm{~nm}$ with a pulse duration of $\tau_{L} \sim$ $120 \mathrm{fs}$ FWHM. Shorter wavelengths are obtained by performing harmonic generation in a nonlinear crystal or in a rare gas (HHG) [17]. The seed used in the current experiment is the second harmonic of the laser, obtained through frequency doubling in a $\beta$-Barium borate crystal, and can produce as much as $150 \mu \mathrm{J}$ of $\lambda_{\text {seed }}=400 \mathrm{~nm}$ ultrashort laser pulses. These pulses are more than sufficient for the FEL to reach saturation and induce superradiance. The saturation energy is indeed roughly given by $E_{\text {sat }}=\rho I_{\text {peak }} E_{B} \tau_{L} / e_{0} \approx 3.5 \mu \mathrm{J}$, with the electron charge

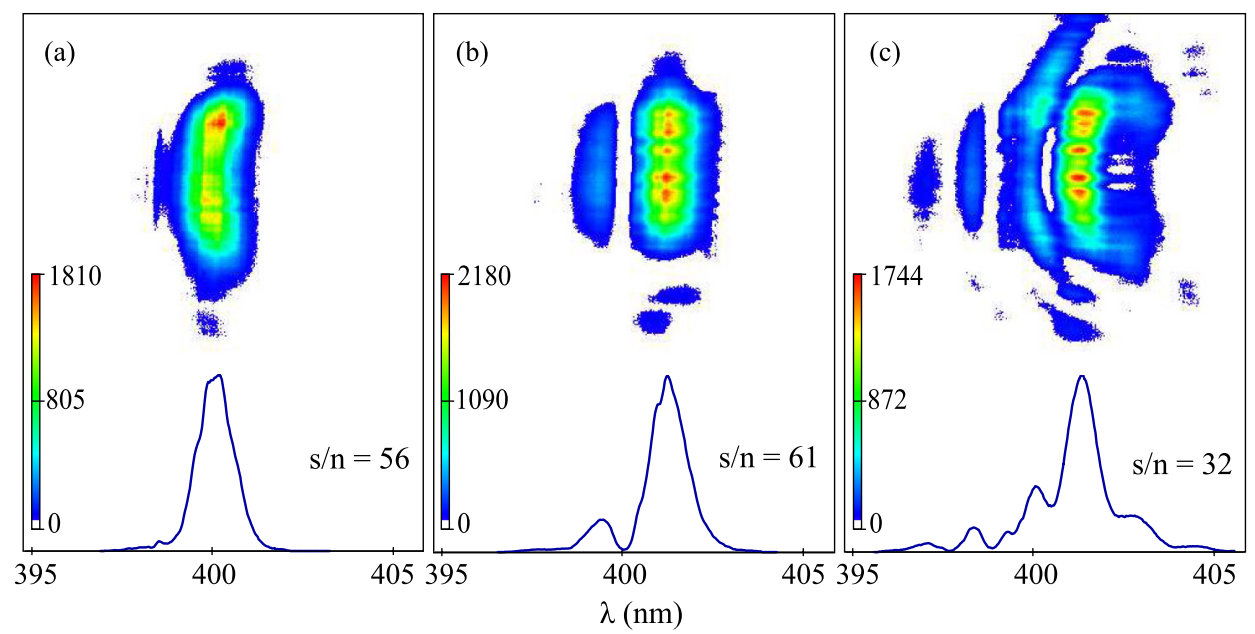

FIG. 2 (color online). Single shot spectra-spatial measurements carried out varying the seed energy $E_{s}:\left(\right.$ a) $E_{s}=1.2 \pm 0.4 \mathrm{~nJ}$, (b) $E_{s}=0.25 \pm 0.06 \mu \mathrm{J}$, and (c) $E_{s}=2.0 \pm 0.5 \mu \mathrm{J}$. The electron beam characteristics correspond to case (A) in Table I. The vertical axis represents the vertical position on the input spectrometer slit $(6.5 \mathrm{~mm})$. Spectra were obtained by means of an in-vacuum spectrometer [30] (normal incidence grating imaging the variable entrance slit on an UV grade CCD camera, Versarray, 1300BPrinceton Instruments). The color intensity (lower left color bar) is expressed in $[(\mathrm{nJ} / \mathrm{mm}) / \mathrm{nm}]$. A cleaning procedure is applied to the images to remove the background noise. The signal to noise ratio $(s / n)$ prior to cleaning is reported on each plot. The average pulse energy $\langle E\rangle$ and rms energy fluctuations $\sigma_{E}$ in the three conditions are given by (a) $\langle E\rangle=3.6 \pm 2.1 \mu \mathrm{J}$, (b) $\langle E\rangle=7.2 \pm 2.0 \mu \mathrm{J}$, and (c) $\langle E\rangle=6.5 \pm 1.3 \mu \mathrm{J}$. The displayed spectra pulse energies are (a) $E=5.3 \mu \mathrm{J}$, (b) $E=8.0 \mu \mathrm{J}$, and (c) $E=8.4 \mu \mathrm{J}$. 
$e_{0}$, the Pierce parameter $\rho$ [11], and the other parameters defined in Table I.

Single shot spectra of the seeded FEL amplifier at increasing seed energies are shown in Fig. 2. While at low seed energy [Fig. 2(a)], the amplified signal has approximately the same spectral distribution as the seed [20], at higher energies [(b) and (c)], a sideband structure appears as an indication of saturation. In all the cases, the FEL output energy is of the same order of magnitude and larger than the expected saturation energy. The weak horizontal structure is associated to the interference generated by the reflection on the vacuum chamber.

A further increase of the seed energy $(9 \mu \mathrm{J})$ leads to the spectrum of the amplified signal, as shown in Fig. 3 (1st), obtained with the beam parameters of Table I, (B). The spectra of the harmonics $\lambda_{n}=\lambda_{\text {seed }} / n$ for $n=2, \ldots, 11$ up to $\lambda_{11} \sim 36 \mathrm{~nm}$ were measured in the same conditions [Fig. 3 (2nd-11th)]. The spectrum of the fundamental reveals a pattern organized in regular fringes of period $\delta \lambda \approx 0.5 \mathrm{~nm}$. This structure, preserved when averaging the spectrum over 100 acquisitions, is distinguishable in shape and separation from the typical self amplified spontaneous emission spiking, where the number of peaks and their positions change randomly from shot to shot. The sideband pattern may be attributed to the interference between the spike in the leading edge of the pulse (see Fig. 1) with radiation produced by overbunched electrons in the pulse tail. The spectral periodicity can provide an indication of the total length $\delta \zeta$ of the wake behind the spike and suggests a longitudinal structure on a scale $\delta \zeta=$ $\lambda_{r}^{2} / \delta \lambda \sim 300 \mu \mathrm{m}$. The wake is associated with the slippage of the light over fresh electrons, and its length grows with the distance covered by the pulse along the undulator in superradiance, when the radiation shifts at the speed of light in-vacuum, over the moving electron bunch. The separations in the spectra in Figs. 2(b) and 2(c) are $\delta \lambda \sim$ $1.8 \mathrm{~nm}$ and $1.3 \mathrm{~nm}$, respectively, so the estimated wake length is $\delta \zeta \sim 90 \mu \mathrm{m}$ in (b) and $\delta \zeta \sim 123 \mu \mathrm{m}$ in (c).
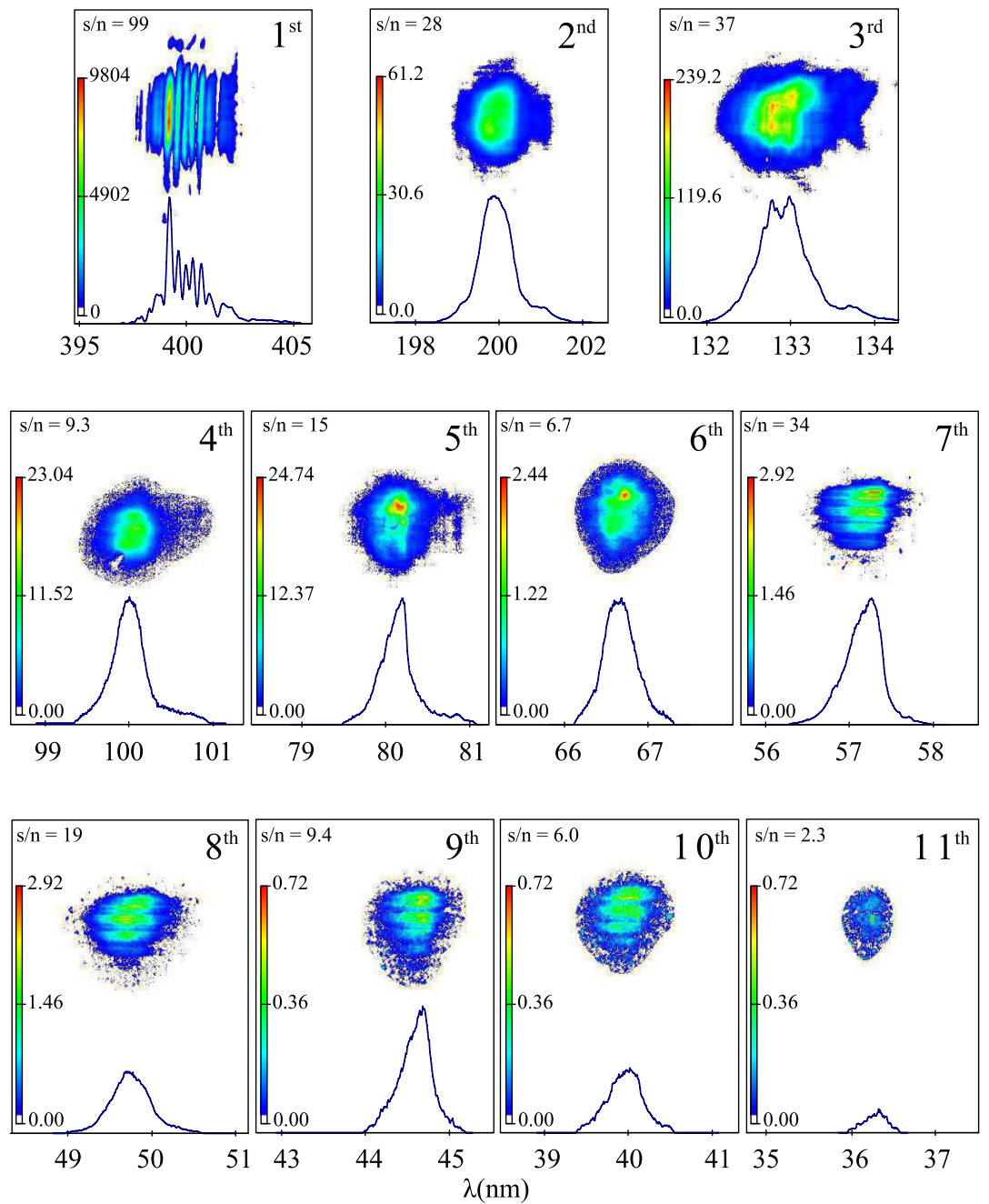

FIG. 3 (color online). Same as in Fig. 2, the spectra of the harmonics from the 1st to the 11th. Beam data of Table I, case (B). Spectra from the 7 th to the 11th harmonics have been acquired, interposing an Al filter to improve the $s / n$ ratio (upper left corner in each image). 


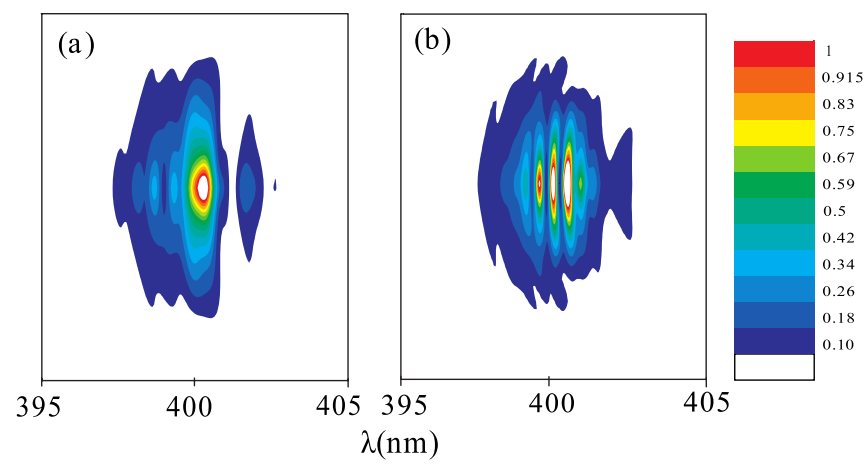

FIG. 4 (color online). (a) GENESIS 1.3 [31] data post processed through a procedure resembling the slit, grating, and CCD of the spectra detection system. The simulation done with the parameters in Table I, case (B). The pulse energy is $E=18 \mu \mathrm{J}$, to be compared with the value observed in the experiment $(E=$ $20 \mu \mathrm{J} \pm 6 \mu \mathrm{J}$ ). (b) Same data as in (a), but with the addition of a pedestal in the laser seed with an intensity $10^{-5}$ smaller than the main pulse.

The structure in the spectrum is well reproduced by GENESIS 1.3 simulations, as shown in Figs. 4(a) and 4(b). The fringes present a periodicity of about $1 \mathrm{~nm}$ corresponding to a wake length $\delta \zeta \sim 160 \mu \mathrm{m}$, while the total slippage length is $N \lambda_{r} \sim 180 \mu \mathrm{m}$, where $N=450$ is the number of periods of the whole SPARC undulator. The higher periodicity observed in the experiment could be attributed to the presence of a small pedestal in the seed laser, amplified in the exponential regime and approaching the onset of saturation at the end of the undulator, only when the seed energy exceeds a few microjoules. This behavior is reproduced in the GENESIS 1.3 simulation in Fig. 4(b), where we added to the seed a replica $10^{-5}$ times lower than the main pulse, delayed $\sim 1 \mathrm{ps}$ in time.

The harmonic spectra (Fig. 3) up to the 6th order correspond to single shot acquisitions. The 7 th-11th spectra are acquired by interposing an aluminum $(\mathrm{Al})$ filter in order to reduce the background noise due to lower order harmonics at the zero order of the grating. The horizontal lines are introduced by a mesh sustaining the $\mathrm{Al}$ layer. The absorption from the $\mathrm{Al}$ layer and the low intensity generated at these harmonics required to integrate on an acquisition time of $10 \mathrm{~s}$. The pattern observed on the fundamental cannot be detected on the higher order harmonics. However, for the 7th-11th harmonics, we cannot exclude the presence of a structure because the measurement suffers a limitation in spectral resolution. The relative harmonic spectral widths, represented in Fig. 5 together with simulations, are almost independent from the harmonic order. This is consistent with the picture of the superradiant pulse represented in Fig. 1, showing that the bunching and the harmonic emission process occur mainly in a limited longitudinal area in the leading edge of the pulse, and that the length of this region diminishes with the harmonic order. The duration of a Fourier transform limited Gaussian pulse with the same spectral width as the second harmonic is about $50 \mathrm{fs}$ (fwhm), with higher harmonics scaling accordingly.

The corresponding harmonics output energies are plotted in Fig. 6 (black symbols). The measured pulse energy at the fundamental, averaged over 100 shots, is $E=20 \mu \mathrm{J} \pm$ $6 \mu \mathrm{J}$, which is substantially higher than the energy $E_{\text {sat }}$ at the onset of saturation, as expected when the pulse propagates in a superradiant regime over a large portion of the undulator. A striking agreement with simulations done with PERSEO and GENESIS 1.3 (red and blue, respectively) is found on the fundamental, confirming the ability of the existing codes to model superradiance and deep saturation. A good agreement is also found at the odd harmonics up to the highest order. Even harmonics cannot be simulated in PERSEO, while for GENESIS 1.3, a substantial and systematic underestimation is found. The result of the analytical model in [27] is also shown in Fig. 6 [lines (a) and (b)]. It has to be noted that this model provides the harmonic energy at the onset of saturation and neglects the pulse propagation effects, therefore it is not fully suited at describing the FEL superradiant dynamics. However, the ratio between the harmonics is well reproduced, as it is shown by the curve (b), which is normalized to the energy measured at the fundamental.

In this Letter, we report the first experimental measurement of high harmonic generation up to the 11th order in a

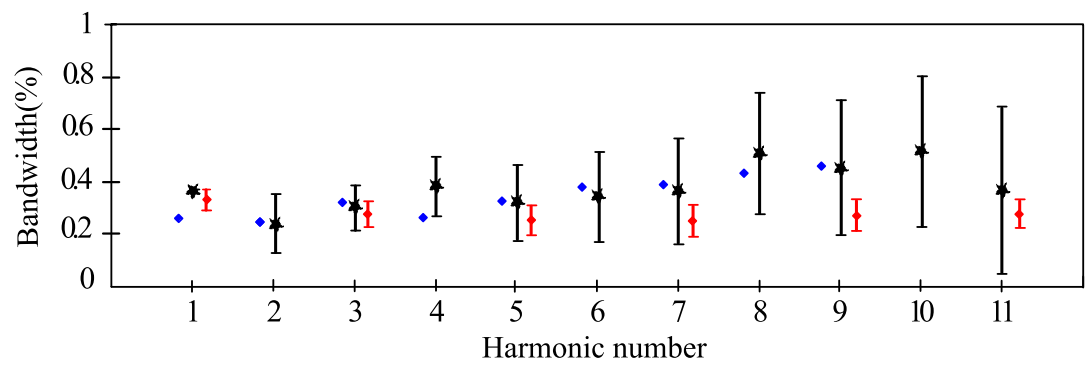

FIG. 5 (color online). Relative rms. bandwidth vs harmonic order for spectra in Fig. 3 (black stars), GENESIS 1.3 simulation (blue rhombus), and PERSEO simulation (red rhombus with error bars). The latter is the result of averaging over 100 simulations where a jitter in the beam energy as given in Table I (A) has been included. 


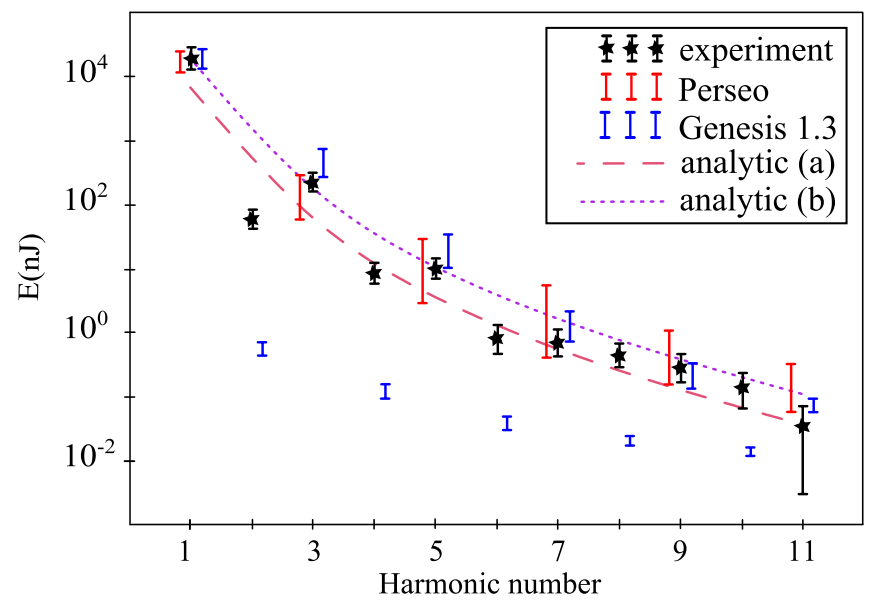

FIG. 6 (color online). Experimental and simulated energy vs harmonics number. Error bars for the experimental data (black stars) represent rms fluctuation combined with error propagation of the calibration factors. Simulations data by PERSEO (at the left of the exp. data) and GENESIS 1.3 (at the right) are the result of averaging over 100 simulations with an rms jitter in the beam energy of $0.27 \mathrm{MeV}$ [Table I case (A)]. The curves represent the predictions of the model in [27] (a) and normalized to the measured energy at the fundamental (b).

FEL amplifier operating in the superradiant regime. The behavior of the single-pass FEL in deep saturation has been analyzed by seeding the amplifier with short laser pulses with peak power of the same order of the FEL saturation power. The pulse energy and bandwidth of the fundamental and higher harmonics have been compared with the values provided by existing codes and analytical models. This measurement provides further insight into the saturated FEL dynamics and harmonic conversion processes. While the generation of high harmonics allows us to extend the FEL operation range, the conversion efficiency at the highest orders can be substantially improved by implementing cascaded FEL schemes [24,28], where saturation has been proposed to simultaneously shorten the pulse length and convert the operation wavelength to a range where $\mathrm{x}$-ray radiation applications demand ultrashort coherent sources.

We wish to acknowledge the entire SPARC team for the long hours spent in the control room, making this work possible. This work has been partially supported by Ministero dell'Istruzione, dell'Universita ' e della Ricerca (DM1834 RIC.4-12-2002) and by the EU sixth framework program, Contract No. 011935 EUROFEL.

*Luca.Giannessi@enea.it

[1] J. Miao, K. O. Hodgson, T. Ishikawa, C. A. Larabell, M. A. LeGros, and Y. Nishino, Proc. Natl. Acad. Sci. U.S.A. 100, 110 (2003).
[2] I. Robinson, I. A. Vartanyants, G. J. Williams, M. A. Pfeifer, and J.A. Pitney, Phys. Rev. Lett. 87, 195505 (2001).

[3] D. Shapiro et al., Proc. Natl. Acad. Sci. U.S.A. 102, 15343 (2005).

[4] K. J. Gaffney and H. N. Chapman, Science 316, 1444 (2007).

[5] P. B. Corkum, Phys. Rev. Lett. 71, 1994 (1993).

[6] T. Brabec and F. Krausz, Rev. Mod. Phys. 72, 545 (2000).

[7] I. Boscolo and V. Stagno, Nuovo Cimento Soc. Ital. Fis. B 58, 267 (1980), .

[8] L. H. Yu et al., Science 289, 932 (2000).

[9] H. Haus, IEEE J. Quantum Electron. 17, 1427 (1981).

[10] G. Dattoli, A. Marino, A. Renieri, and F. Romanelli, IEEE J. Quantum Electron. 17, 1371 (1981).

[11] R. Bonifacio, C. Pellegrini, and L. M. Narducci, Opt. Commun. 50, 373 (1984).

[12] R. Bonifacio, L. De Salvo, P. Pierini, N. Piovella, and C. Pellegrini, Phys. Rev. Lett. 73, 70 (1994).

[13] A. Doyuran et al., Phys. Rev. Lett. 86, 5902 (2001).

[14] R. Prazeres et al., Nucl. Instrum. Methods Phys. Res., Sect. A 304, 72 (1991).

[15] L. H. Yu, L. DiMauro, A. Doyuran, and W. S. Graves et al., Phys. Rev. Lett. 91, 074801 (2003).

[16] T. Togashi et al., Opt. Express 19, 317 (2011).

[17] M. Labat et al., Phys. Rev. Lett. 107, 224801 (2011).

[18] S. Di Mitri et al., Proceedings of the IPAC 2011 TZA01 (2011) (unpublished).

[19] G. Lambert et al., Nature Phys. 4, 296 (2008).

[20] T. Tanikawa, G. Lambert, T. Hara, M. Labat, Y. Tanaka, M. Yabashi, O. Chubarand,, and M. E. Couprie, Europhys. Lett. 94, 34001 (2011).

[21] R. H. Dicke, Phys. Rev. 93, 99 (1954).

[22] R. Bonifacio, L. De Salvo, P. Pierini, and N. Piovella, Nucl. Instrum. Methods Phys. Res., Sect. A 296, 358 (1990).

[23] T. Watanabe, X. J. Wang, J. B. Murphy, J. Rose, Y. Shen,T. Tsang, L. Giannessi, P. Musumeci and S. Reiche, Phys. Rev. Lett. 98, 034802 (2007).

[24] L. Giannessi, P. Musumeci, and S. Spampinati, J. Appl. Phys. 98, 043110 (2005).

[25] L. Giannessi et al., Phys. Rev. ST Accel. Beams 14, 060712 (2011).

[26] M. Quattromini et al., in Proceedings of the 2008 EPAC Conference (2008), p. WEPC124, www.jacow.org.

[27] G. Dattoli and P. L. Ottaviani, J. Appl. Phys. 86, 5331 (1999).

[28] L. Giannessi and P. Musumeci, New J. Phys. 8, 294 (2006).

[29] L. Giannessi, Phys. Rev. ST Accel. Beams 6, 114802 (2003).

[30] L. Poletto, S. Bonora, M. Pascolini, and P. Villoresi, Rev. Sci. Instrum. 75, 4413 (2004).

[31] S.Reiche, Nucl. Instrum. Methods Phys. Res., Sect. A 429, 243 (1999). 